\title{
Numerical investigation of finite-volume effects for the HVP
}

\author{
Peter Boyle ${ }^{1}$, Vera Gülpers ${ }^{2}$, James Harrison ${ }^{2 \star \star}$ Andreas Jüttner $^{2}$, Antonin Portelli ${ }^{1}$, and Christo- \\ pher Sachrajda ${ }^{2}$ \\ ${ }^{1}$ School of Physics and Astronomy, University of Edinburgh, Edinburgh EH9 3JZ, United Kingdom \\ ${ }^{2}$ School of Physics and Astronomy, University of Southampton, Southampton SO17 1BJ, United Kingdom
}

\begin{abstract}
It is important to correct for finite-volume (FV) effects in the presence of QED, since these effects are typically large due to the long range of the electromagnetic interaction. We recently made the first lattice calculation of electromagnetic corrections to the hadronic vacuum polarisation (HVP). For the HVP, an analytical derivation of FV corrections involves a two-loop calculation which has not yet been carried out. We instead calculate the universal FV corrections numerically, using lattice scalar QED as an effective theory. We show that this method gives agreement with known analytical results for scalar mass FV effects, before applying it to calculate FV corrections for the HVP. This method for numerical calculation of FV effects is also widely applicable to quantities beyond the HVP.
\end{abstract}

\section{Introduction}

Currently, there is a discrepancy greater than $3 \sigma$ between Standard Model predictions and experimental measurements of the muon $g-2$ [1]. This is a tantalising hint of possible new physics, and new experiments at Fermilab and J-PARC are set to reduce the experimental error in the measurement $[2,3]$. The largest source of theoretical error in the muon $g-2$ prediction comes from the hadronic vacuum polarisation (HVP) contribution.

In an effort to reduce the theoretical uncertainty in the HVP, lattice calculations of QED corrections to the HVP are in progress. We have made an exploratory lattice calculation of QED corrections to the HVP [4-6], and there is an ongoing effort to calculate these corrections at the physical point [7]. Independently, a calculation of QED corrections for strange and charm contributions to the HVP has also been made [8].

The mass gap in QCD ensures that effects due to the finite volume of the lattice fall off exponentially with the spatial extent $L$ of the lattice, provided $L$ is significantly larger than the inverse pion mass [9]. In QED, however, there is no mass gap and the massless photon ensures that electromagnetism is a long-range interaction. Finite volume (FV) effects for QED are therefore much larger than those for QCD, typically scaling with inverse powers of $L$ rather than exponentially [10], and they must be taken into account in any physical calculation of QED effects on the lattice.

FV effects arise from long-distance properties, and do not therefore depend strongly on UV properties of the system. Leading FV effects can therefore be studied using low-energy effective field

${ }^{\star}$ Speaker, e-mail: J.Harrison@ soton.ac.uk 
theories in which hadrons are treated as point particles. FV corrections have been calculated analytically for hadron masses $[10,11]$ and leptonic decay rates [12], within the $\mathrm{QED}_{L}$ zero-mode subtraction scheme [13].

In this work, we use computationally cheap lattice scalar QED simulations for a numerical investigation of QED FV effects for the HVP. In section 2 of this proceedings, our strategy for calculating FV effects through lattice scalar QED calculations is introduced. Meson mass FV effects obtained using this method are validated against known results in section 3, and preliminary results for HVP finite volume effects are shown in section 4.

\section{Numerical simulations of lattice scalar QED}

The strategy for numerical calculation of finite volume effects from lattice scalar QED is straightforward: expectation values are calculated from the discretised scalar QED path integral, and this is repeated for several lattice volumes. FV corrections can be obtained numerically by assuming an ansatz for the dependence of the expectation values on the spatial extent $L$ of the lattice, and extracting coefficients through a fit to the lattice data points.

This method can be applied to study the QED finite volume behaviour of any hadronic quantity for which scalar QED is a valid effective field theory. Here, we validate the method by comparing to known analytical results for pseudoscalar mesons, before turning our attention to the HVP.

\subsection{Lattice scalar QED action}

We define the discretised scalar QED action as

$$
S[\phi, A]=S_{\phi}[\phi, A]+S_{\gamma}[A],
$$

with non-compact $U(1)$ gauge action

$$
S_{\gamma}[A]=\frac{1}{4} \sum_{x} \sum_{\mu, v}\left(\partial_{\mu} A_{v}(x)-\partial_{\nu} A_{\mu}(x)\right)^{2},
$$

for an electromagnetic vector potential $A_{\mu}(x)$ and forward derivative $\partial_{\mu}$, and scalar action

$$
S_{\phi}[\phi, A]=\frac{a^{4}}{2} \sum_{x} \phi^{*}(x) \Delta \phi(x), \quad \Delta=-\sum_{\mu} D_{\mu}^{*} D_{\mu}+m^{2},
$$

where $\phi(x)$ is a complex scalar field. The covariant derivative is defined as

$$
\begin{aligned}
& D_{\mu} f(x)=a^{-1}\left[U_{\mu}(x) f(x+a \hat{\mu})-f(x)\right], \\
& D_{\mu}^{*} f(x)=a^{-1}\left[f(x)-U_{\mu}^{\dagger}(x-a \hat{\mu}) f(x-a \hat{\mu})\right],
\end{aligned}
$$

with $U(1)$ gauge link $U_{\mu}(x) \equiv e^{i q a A_{\mu}(x)}$ for a scalar with electromagnetic charge $q$.

We work in the electro-quenched theory, in which we set $\sqrt{\operatorname{det}\left(\Delta^{-1}\right)}=1$ in the path integral, so that $U(1)$ gauge field configurations can be generated independently of the scalar field. Generation of the photon fields follows the method of [14], and the photon zero-mode is subtracted in the $\mathrm{QED}_{L}$ scheme [13]. 


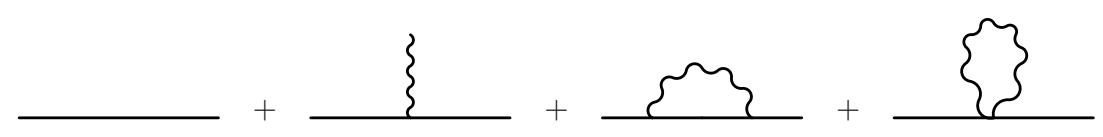

Figure 1. The four diagrams which correspond to the expansion to $O(\alpha)$ of the charged scalar propagator (equation (12)).

\subsection{Inverting the scalar propagator}

Calculation of the scalar propagator $\Delta^{-1}$ could be done using the CG algorithm, but we instead use a perturbative method, making use of the Fast Fourier Transform (FFT) algorithm. The motivation for using this method is twofold. Firstly, this allows a perturbative treatment of the QED effects up to fixed order, which facilitates comparison with analytical calculations. Secondly, the computational cost of the FFT is independent of the input mass, so we expect this method to be more efficient than CG for small masses.

Using the translation operator

$$
\tau_{\mu} f(x)=f(x+a \hat{\mu}),
$$

the operator $\Delta$ can be written as

$$
\Delta=a^{-2}\left[2-e^{i q a A_{\mu}} \tau_{\mu}-\tau_{-\mu} e^{-i q a A_{\mu}}\right]+m^{2} .
$$

Expanding in powers of the electromagnetic charge $q$ gives

$$
\begin{aligned}
\Delta & =\Delta_{0}+q \Delta_{1}+q^{2} \Delta_{2}+O\left(q^{3}\right), \\
\Delta_{0} & =-\sum_{\mu} \partial_{\mu}^{*} \partial_{\mu}+m^{2}, \\
\Delta_{1} & =-i a^{-1} \sum_{\mu}\left(A_{\mu} \tau_{\mu}-\tau_{-\mu} A_{\mu}\right), \\
\Delta_{2} & =\frac{1}{2} \sum_{\mu}\left(A_{\mu}^{2} \tau_{\mu}+\tau_{-\mu} A_{\mu}^{2}\right) .
\end{aligned}
$$

We can then expand the scalar propagator:

$$
\Delta^{-1}=\Delta_{0}^{-1}-q \Delta_{0}^{-1} \Delta_{1} \Delta_{0}^{-1}+q^{2} \Delta_{0}^{-1} \Delta_{1} \Delta_{0}^{-1} \Delta_{1} \Delta_{0}^{-1}-q^{2} \Delta_{0}^{-1} \Delta_{2} \Delta_{0}^{-1}+O\left(q^{3}\right) .
$$

The four terms in this expansion to $O(\alpha)$ correspond to the four diagrams in Figure 1. The first term is the free scalar propagator, the second is a scalar propagator with a single photon vertex, and the final two terms are $O(\alpha)$ corrections which we refer to as the "sunset" and "tadpole" diagrams respectively.

Defining the Fourier transform of a field $f$ on the lattice $\Lambda$ as

$$
(\mathcal{F} f)(k)=a^{4} \sum_{x \in \Lambda} f(x) e^{-i k \cdot x},
$$

the free scalar propagator $\Delta_{0}^{-1}$ and the operators $\Delta_{1}$ and $\Delta_{2}$ can be expressed as 


$$
\begin{aligned}
\Delta_{0}^{-1} & =\mathcal{F}^{-1} \frac{1}{\hat{k}^{2}+m^{2}} \mathcal{F}, \\
\Delta_{1} & =-i a^{-1} \sum_{\mu}\left[A_{\mu} \mathcal{F}^{-1} e^{i a k_{\mu}} \mathcal{F}-\mathcal{F}^{-1} e^{-i a k_{\mu}} \mathcal{F} A_{\mu}\right], \\
\Delta_{2} & =\frac{1}{2} \sum_{\mu}\left[A_{\mu}^{2} \mathcal{F}^{-1} e^{i a k_{\mu}} \mathcal{F}+\mathcal{F}^{-1} e^{-i a k_{\mu}} \mathcal{F} A_{\mu}^{2}\right] .
\end{aligned}
$$

Using equations (12), (14), (15) and (16), along with stochastic $U(1)$ gauge fields $A_{\mu}$, the calculation of the charged scalar propagator to $O(\alpha)$ requires 44 Fourier transforms, which can be computed using the FFT algorithm.

This propagator inversion method has been implemented in Grid, a new software framework developed by members of the RBC/UKQCD collaboration [15]. The design of Grid allows the construction of observables from these propagators to be easily implemented while providing good performance across a range of computational architectures.

\section{Meson mass finite volume effects}

In order to validate this numerical approach to the calculation of FV corrections, it is useful to compare with known results. One quantity for which the FV corrections are known is the mass of a charged scalar particle, for which the volume dependence in the $\mathrm{QED}_{L}$ scheme is [10]

$$
m^{2}(L) \sim m_{\infty}^{2}-\frac{q^{2}}{4 \pi} \frac{\kappa}{L}\left(m_{0}+\frac{2}{L}\right)+O\left(e^{-m L}\right), \quad \kappa=2.837297(1),
$$

where $q$ is the EM charge of the scalar, $m_{0}$ is the bare mass and $m_{\infty}$ is the mass in infinite volume. This relation gives the universal FV corrections to the mass of all spin- 0 particles, including composite particles such as the pseudoscalar mesons, up to structure-dependent terms which have been shown to be no larger than $O\left(\alpha / L^{3}\right)[11]$.

\subsection{Extracting the scalar mass from lattice data}

To calculate the scalar mass, we start by computing the point-to-all scalar propagator from a point source at the origin:

$$
S(0 \mid x)=\Delta_{y x}^{-1} \delta(y=0) .
$$

We define an effective mass correction from the zero-spatial-momentum component of the scalar propagator, $S(t)=\sum_{\vec{x}} S(0 \mid x)[5]$ :

$$
\delta m_{\mathrm{eff}}(t)=\frac{\frac{S(t)}{S_{0}(t)}-\frac{S(t+1)}{S_{0}(t+1)}}{\left(\frac{T}{2}-t\right) \tanh \left(m_{0}\left(\frac{T}{2}-t\right)\right)-\left(\frac{T}{2}-(t+1)\right) \tanh \left(m_{0}\left(\frac{T}{2}-(t+1)\right)\right)},
$$

where $T$ is the time extent of the lattice and $S_{0}(t)$ is the free scalar propagator, and the mass correction $\delta m(L)$ is extracted from a constant fit to the effective mass plateau. The squared mass up to $O(\alpha)$ is constructed from $\delta m(L)$ :

$$
m^{2}(L)=m_{0}^{2}+2 m_{0} \delta m(L)
$$




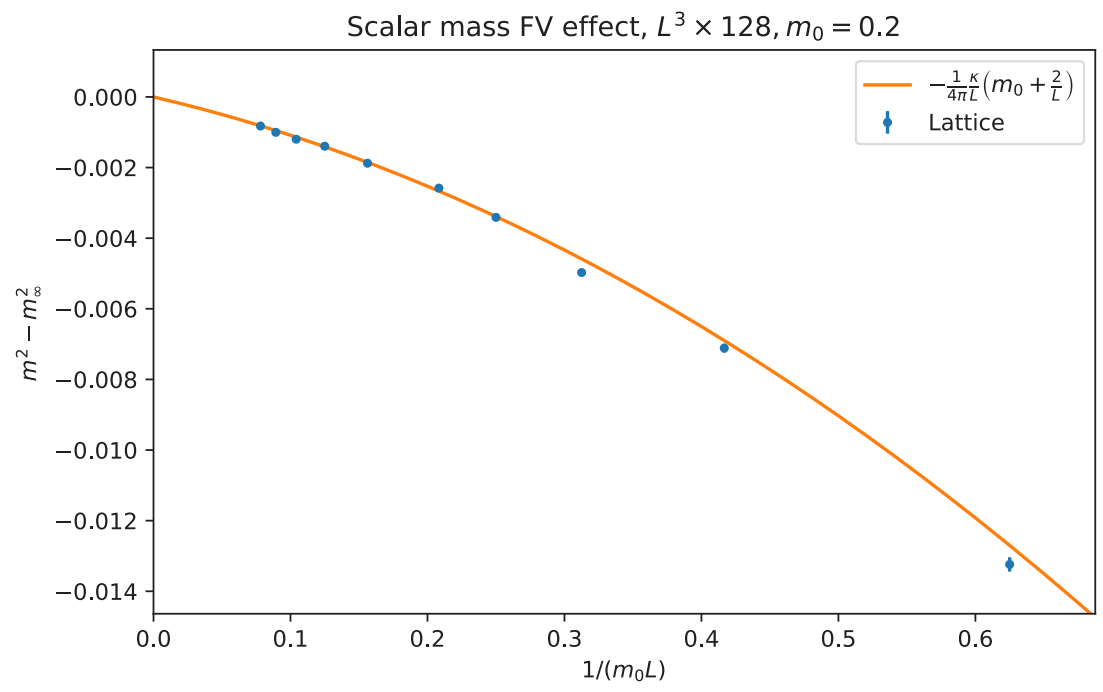

Figure 2. Comparison of lattice scalar mass with the analytical expression in equation (17).

\subsection{Results}

The scalar mass has been calculated on a $L^{3} \times 128$ lattice, for a range of values of $L$, with bare mass $a m_{0}=0.2 .100 U(1)$ gauge configurations were used per volume.

In Figure 2, our lattice data are compared to the analytical expression in equation (17). The infinite-volume squared mass is calculated analytically and subtracted from the data. The chi-squared statistic for the goodness-of-fit of the analytical prediction to the data points with $m_{0} L \geq 4$ is $\chi^{2} /$ d.o.f. $=0.27$, indicating that the lattice data match the expected FV behaviour very well. The agreement with the analytical expression is less good for smaller volumes, indicating that the exponentially suppressed terms excluded from equation (17) are non-negligible at this level.

The precision achieved here from 100 configurations is impressive. The computational cost of generating these results was small: for the largest volume $(L=64)$, the total computation time for 100 configurations on a single Knights Landing processor was approximately 24 hours. These observations confirm that our numerical method for calculating FV effects can provide good precision with limited time and computational resources.

\section{HVP finite volume effects}

We expect the FV behaviour of the HVP to be dominated by the lowest-energy state, which is the two-pion state. The leading FV corrections can therefore be determined from the FV behaviour of the scalar QED vacuum polarisation (SVP) (Figure 3), which can be investigated numerically in the framework outlined above.

We calculate the SVP from the conserved vector two-point correlation function as follows:

$$
\Pi\left(\hat{Q}^{2}\right)=\frac{1}{3} a^{4} \sum_{j} \sum_{x} C_{j j}(x)\left(\frac{e^{-i Q t}-1}{\hat{Q}^{2}}\right), \quad C_{\mu \nu}(x)=\left\langle V_{\mu}(x) V_{v}(0)\right\rangle,
$$




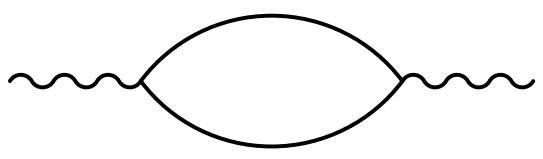

Figure 3. The scalar QED vacuum polarisation (SVP). Solid lines represent scalars.

where $V_{\mu}(x)$ is the conserved vector current derived from the scalar QED action in equation (1):

$$
V_{\mu}(x)=a^{2}\left[\phi^{*}(x) e^{i q a A_{\mu}(x)} \phi(x+a \hat{\mu})-\phi^{*}(x+a \hat{\mu}) e^{-i q a A_{\mu}(x)} \phi(x)\right]
$$

From equations (21) and (22) we find

$$
C_{\mu \nu}(x)=\left\langle 2 \mathfrak{R}\left[S^{\dagger}(a \hat{v} \mid x) U_{\mu}(x) S(0 \mid x+a \hat{\mu}) U_{v}(0)-S^{\dagger}(a \hat{v} \mid x+a \hat{\mu}) U_{\mu}^{\dagger}(x) S(0 \mid x) U_{v}(0)\right]\right\rangle_{\gamma}
$$

where $\langle\cdot\rangle_{\gamma}$ denotes the average over $U(1)$ gauge configurations.

The gauge links $U_{\mu}(x) \equiv e^{i q a A_{\mu}(x)}$ which appear in the conserved vector current at the source and sink can be expanded in powers of the charge $q$ :

$$
U_{\mu}(x)=1+i q a A_{\mu}(x)-\frac{1}{2} q^{2} a^{2} A_{\mu}^{2}(x)+O\left(q^{3}\right) .
$$

Combining equations (12), (23) and (24), there are 12 diagrams which give an $O(\alpha)$ contribution to the SVP: a sunset or tadpole on either scalar propagator, a photon exchanged between the two scalar propagators, and seven diagrams in which the photon attaches to the source and/or sink due to the expansion of the gauge link in the conserved current [5].

\subsection{Preliminary results}

In this section we show some preliminary results for HVP FV effects, with the same parameters as were used for the scalar mass in Section 3.2.

FV effects for the free SVP are expected to be exponential. To check this, we assume an ansatz

$$
\Pi_{0}(L)=c_{0}\left(1-c_{1} e^{-c_{2} m_{0} L}\right) .
$$

At the smallest non-zero lattice momentum $(Q=(2 \pi / T))$ we find that this ansatz describes the data well with $c_{0}=6.32 \times 10^{-3}, c_{1}=1.57$ and $c_{2}=0.90$, as shown in Figure 4 .

Figure 5 shows the $O(\alpha)$ correction to the SVP at $Q=(2 \pi / T)$. One could fit a polynomial ansatz in $1 / L$ to these data points, to obtain a numerical estimate for the coefficients of the FV corrections, but we do not attempt such a fit here. An analytical calculation of these FV corrections is in progress, and the results presented here will be compared directly with those from the analytical calculation.

\section{Conclusions}

We introduce a method for the efficient numerical calculation of universal QED finite volume corrections to hadronic observables, using lattice simulations of scalar QED as an effective field theory. The technique is applied to the calculation of FV corrections to the mass of a scalar particle, and results are found to be in agreement with the known analytical FV behaviour of the scalar mass. 


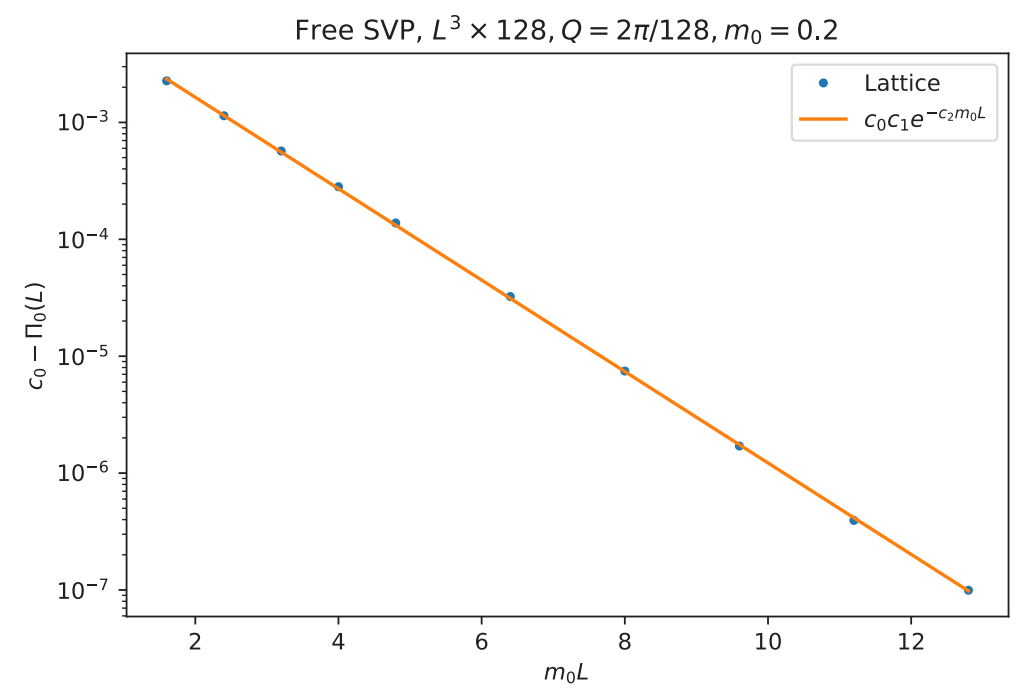

Figure 4. Finite-volume behaviour of the free SVP form factor. The orange line is a fit of the exponential ansatz in equation (25) to the lattice data, with parameters $c_{0}=6.32 \times 10^{-3}, c_{1}=1.57$ and $c_{2}=0.90$.

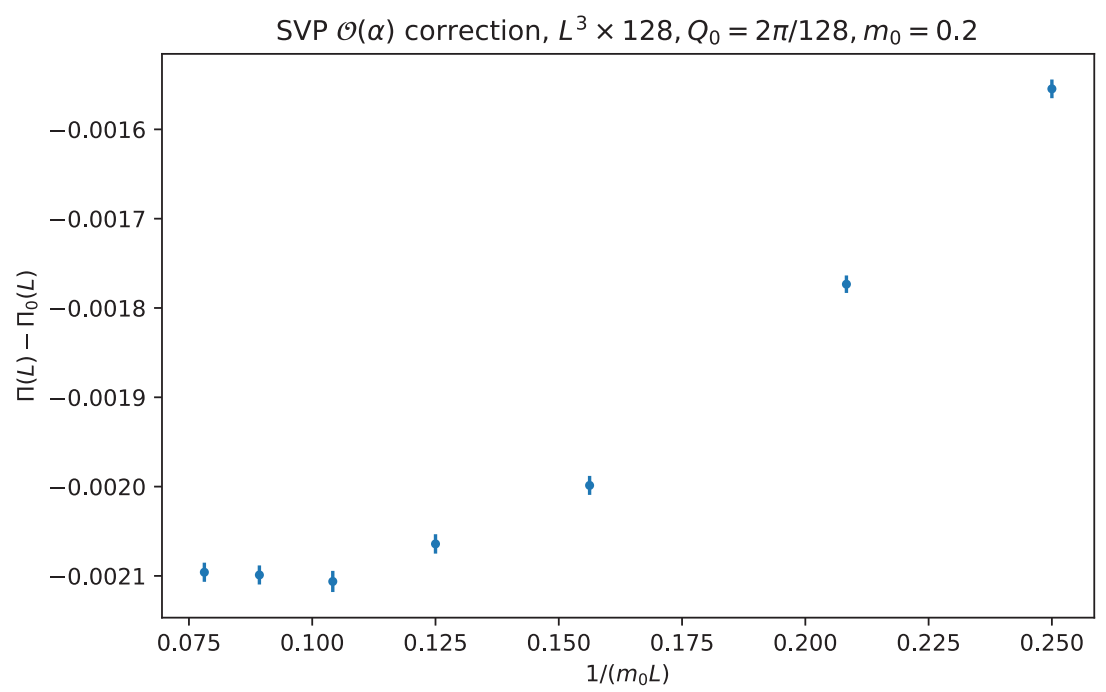

Figure 5. Finite-volume behaviour of the $O(\alpha)$ correction to the SVP form factor for seven volumes with $m_{0} L \geq$ 4. 
We also apply this method to the calculation of QED FV corrections to the HVP. FV effects for the free vacuum polarisation (i.e. without QED) are found to be exponentially suppressed with the lattice size $L$, as expected. We have made a preliminary calculation of the HVP FV effects in the presence of QED, which will be used to cross-check an analytical calculation of these effects.

This method is widely applicable for calculating universal QED FV corrections to a range of hadronic quantities, and we find that the method can provide good precision with limited computational resources. The method has been implemented in Grid [15], so that its application to new observables should be straightforward.

\section{Acknowledgements}

This work has received funding from the STFC Grant ST/L000296/1, the EPSRC Centre for Doctoral Training in Next Generation Computational Modelling grant EP/L015382/1 and from the European Research Council under the European Union's Seventh Framework Programme (FP7/20072013) / ERC Grant agreement 279757. P.A.B. and A.P. are supported in part by UK STFC grant ST/L000458/1.

\section{References}

[1] C. Patrignani et al. (Particle Data Group), Chin. Phys. C40, 100001 (2016)

[2] R. Carey, K. Lynch, J. Miller, B. Roberts, W. Morse, Y. Semertzides, V. Druzhinin, B. Khazin, I. Koop, I. Logashenko et al., Tech. rep., Fermi National Accelerator Laboratory (FNAL), Batavia, IL (United States) (2009), http://www.osti.gov/servlets/purl/ 952029-2TyrgK/

[3] M. Otani, JPS Conf. Proc. 8, 025008 (2015)

[4] P. Boyle, V. Gülpers, J. Harrison, A. Jüttner, A. Portelli, C. Sachrajda, PoS LATTICE2016, 172 (2016), 1612.05962

[5] P. Boyle, V. Gülpers, J. Harrison, A. Jüttner, C. Lehner, A. Portelli, C.T. Sachrajda, JHEP 09, 153 (2017), 1706.05293

[6] V. Gülpers et al. (RBC/UKQCD), Isospin Breaking Corrections to the HVP with Domain Wall Fermions, in Proceedings, 35th International Symposium on Lattice Field Theory (Lattice2017): Granada, Spain, to appear in EPJ Web Conf.

[7] C. Lehner (RBC/UKQCD), A precise determination of the HVP contribution to the muon anomalous magnetic moment from lattice QCD, in Proceedings, 35th International Symposium on Lattice Field Theory (Lattice2017): Granada, Spain, to appear in EPJ Web Conf.

[8] D. Giusti, V. Lubicz, G. Martinelli, F. Sanfilippo, S. Simula (2017), 1707.03019

[9] M. Lüscher, Communications in Mathematical Physics 104, 177 (1986)

[10] S. Borsanyi, S. Durr, Z. Fodor, C. Hoelbling, S.D. Katz, S. Krieg, L. Lellouch, T. Lippert, A. Portelli, K.K. Szabo et al., Science 347, 1452 (2015)

[11] Z. Davoudi, M.J. Savage, Physical Review D 90, 054503 (2014)

[12] V. Lubicz, G. Martinelli, C.T. Sachrajda, F. Sanfilippo, S. Simula, N. Tantalo (2016), 1611.08497

[13] S. Uno, M. Hayakawa, Progress of Theoretical Physics 120, 413 (2008)

[14] A. Duncan, E. Eichten, H. Thacker, Physical Review Letters 76, 3894 (1996)

[15] P. Boyle, A. Yamaguchi, G. Cossu, A. Portelli (2015), 1512.03487 